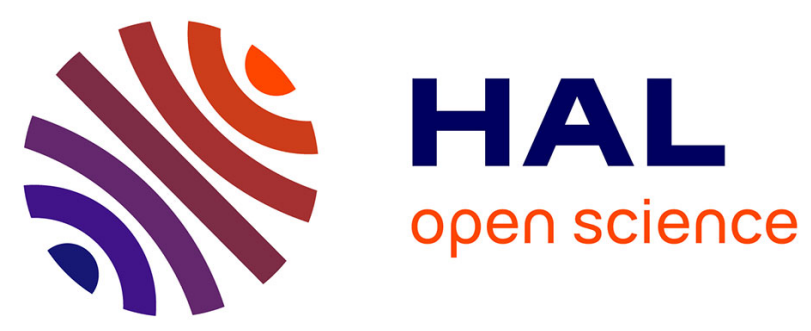

\title{
CHEMICAL VAPOR PRECIPITATION OF SUBMICRON TITANIUM NITRIDE POWDER
}

J. Dekker, P.J. van Der Put, R. Nieuwenhuis, H. Veringa, J. Schoonman

\section{To cite this version:}

J. Dekker, P.J. van Der Put, R. Nieuwenhuis, H. Veringa, J. Schoonman. CHEMICAL VAPOR PRECIPITATION OF SUBMICRON TITANIUM NITRIDE POWDER. Journal de Physique IV Proceedings, 1991, 02 (C2), pp.C2-593-C2-599. 10.1051/jp4:1991271 . jpa-00249861

\section{HAL Id: jpa-00249861 https://hal.science/jpa-00249861}

Submitted on 1 Jan 1991

HAL is a multi-disciplinary open access archive for the deposit and dissemination of scientific research documents, whether they are published or not. The documents may come from teaching and research institutions in France or abroad, or from public or private research centers.
L'archive ouverte pluridisciplinaire HAL, est destinée au dépôt et à la diffusion de documents scientifiques de niveau recherche, publiés ou non, émanant des établissements d'enseignement et de recherche français ou étrangers, des laboratoires publics ou privés. 
Colloque C2, suppl. au Journal de Physique II, Vol. 1, septembre 1991

\title{
CHEMICAL VAPOR PRECIPITATION OF SUBMICRON TITANIUM NITRIDE POWDER
}

\author{
J.P. DEKKER* , P.J. VAN DER PUT ${ }^{* *}$, R.R. NIEUWENHUIS ${ }^{*}$, \\ H.J. VERINGA ${ }^{*}$ and J. SCHOONMAN* * \\ "Netherlands Energy Research Foundation ECN, Westerduinweg 3. \\ P.O. Box 1, NL-1755 ZG Petten, The Netherlands \\ ** Delft University of Technology, Laboratory for Inorganic \\ Chemistry, Julianalaan 136, NL-2628 BL, The Netherlands
}

\begin{abstract}
The synthesis of submicron titanium nitride powder by Chemical vapor Precipitation and the characteristics of primary particles are presented. The properties of the powder formed are of prime importance to the Particle Precipitation aided Chemical Vapor Deposition process. The dependence of the particle size on the synthesis parameters and the differences in powder characteristics are discussed.
\end{abstract}

\section{INTRODUCTION.}

Convential CVD is a valuable method to produce thin dense coatings but deposition rates are low. If gas phase concentrations are increased in order to increase the deposition rate, homogeneous nucleation may occur at the critical supersaturation. Thus aerosol formation limits the maximum deposition rate. This powder formation however can be used to our advantage to increase the deposition rate of layers and to control the porosity of the layer.

In Particle Precipitation aided Chemical Vapor Deposition (PP-CVD) particles formed by nucleation in the gas phase are deposited on a cooled substrate. This precipitation is induced by thermophoresis [1] which is diffusion in a thermal gradient due to the differences in the kinetic energy of gas molecules which cause the particles to move from the warmer to the colder side. simultaneously the pores between the precipitated particles should be partly or entirely filled by heterogeneous reaction, i.e. Chemical vapor Infiltration (CVI).

This principle was first used in the Modified CVD process to deposit doped silica in glass fibres [2]. The PP-CVD process has been introduced by Komijama et al. [3]. They have obtained fast growth rates of titanium oxide, zirconium oxide and aluminium nitride layers. The layers varied from a dense to a highly porous structure depending on experimental conditions and substrate position in the reactor.

The synthesis of submicron powder is of prime importance to PP-CVD. To obtain a uniform layer the powder has to be monodisperse and not agglomerated. Kato et al. have studied the chemical vapor Precipitation (CVP) of titanium nitride from titanium tetrachloride, ammonia and hydrogen [4]. They concluded that the reactants have to be 
introduced separately into the reactor and mixed in the high temperature zone in order to obtain monodisperse powders. We have investigated the synthesis of titanium nitride powder from titanium tetrachloride, ammonia and hydrogen to determine the process conditions suitable for PP-CVD.

Particle sizes are obtained by sedimentation Field Flow Fractionation (SF3), Quasi Elastic Light scattering (QELS) and Transmission Electron Microscope (TEM) analysis. In Sedimentation Field Flow Fractionation a powder suspended in water is flowed through a gravitational field in a centrifuge [5]. The particles are exponentially distributed in the field as a result of a counteracting combination of diffusion and gravitation. The parabolic rate gradient in the laminar flow fractionates the particles according to their weight.

Quasi Elastic Light scattering or Photon Correlation spectroscopy measures intensity autocorrelation of laser light scattered by particles in a liquid suspension [6]. The scattered intensity fluctuates as a result of time modulated interference due to Brownian motion of the dispersed particles. The power spectrum of the scattered light allows determination of the hydrodynamic size of the dispersed particles.

\section{EXPERIMENTAL ASPECTS,}

2.1 Synthesis.

A PP-CVD reactor with a conventional gas handling system is used for the synthesis of titanium nitride powder. Purified hydrogen is applied as a carrier gas for titanium tetrachloride and as a diluent. This mixture is introduced into a quartz tube reactor (ID $=50 \mathrm{~mm}, L=900 \mathrm{~mm}$ ) in a three zone furnace. Electronic grade ammonia is introduced into the second zone which was kept constant within $5{ }^{\circ} \mathrm{C}$ over a length of $400 \mathrm{~mm}$. In the third zone a flat hollow substrate support ( $W=20 \mathrm{~mm}$, $\mathrm{L}=30 \mathrm{~mm}, \mathrm{H}=4 \mathrm{~mm})$, cooled on the inside by pressurized air, collects a representative amount of the formed powder on the substrate which consisted of a piece of molybdenum sheet.

The following experimental conditions were varied: reaction temperature from $900 \mathrm{~K}$ to $1300 \mathrm{~K}$, titanium tetrachloride concentration from 0.5 to 2 percent, reactant ratio ammonia over titanium tetrachloride from 1 to 10 and linear gas flow rate (STP) from 0.5 to $1.5 \mathrm{~cm} / \mathrm{sec}$. The process time was 30 minutes and the temperature of the molybdenum substrate and the temperature gradient between gas phase and molybdenum substrate were kept constant at $800 \mathrm{~K}$ and $100 \mathrm{Kcm}^{-1}$ respectively. The experimental deposition conditions were varied around the following standard values: temperature of $1073 \mathrm{~K}$, titanium tetrachloride concentration of 2 percent, reactant ratio ammonia over titanium tetrachloride of 10 and a linear gas flow rate (STP) of $1 \mathrm{~cm} / \mathrm{sec}$.

\subsection{Particle analysis.}

The collected titanium nitride powder was dispersed ultrasonically in an aqueous ammonia solution $(0.002 \mathrm{M})$ and characterized by a Dupont SF3 Sedimentation Field Flow Fractionation analyser and a Coulter N4 Light scattering analyser. The actual fractionation of eluted volumes of liquid started with a rise in extinction of the ultraviolet detector and could clearly be distinguised from the noise. The fraction collected from the SF3 experiments were analysed directly by the QELS method. 
The dark brown titanium nitride powders formed were crystalline in all cases. The weight of the collected powder on the cooled molybdenum substrate as a function of temperature is presented in Figure 1. The apparent activation energy is $98 \pm 10 \mathrm{~kJ} / \mathrm{mole}$ between $900 \mathrm{~K}$ and $1200 \mathrm{~K}$. This is equal to the activation energy of the conversion of reactant because the sedimentation velocity (i.e. the sum of linear gas velocity and thermophoretic velocity) of the particle is independent of their diameter [I].

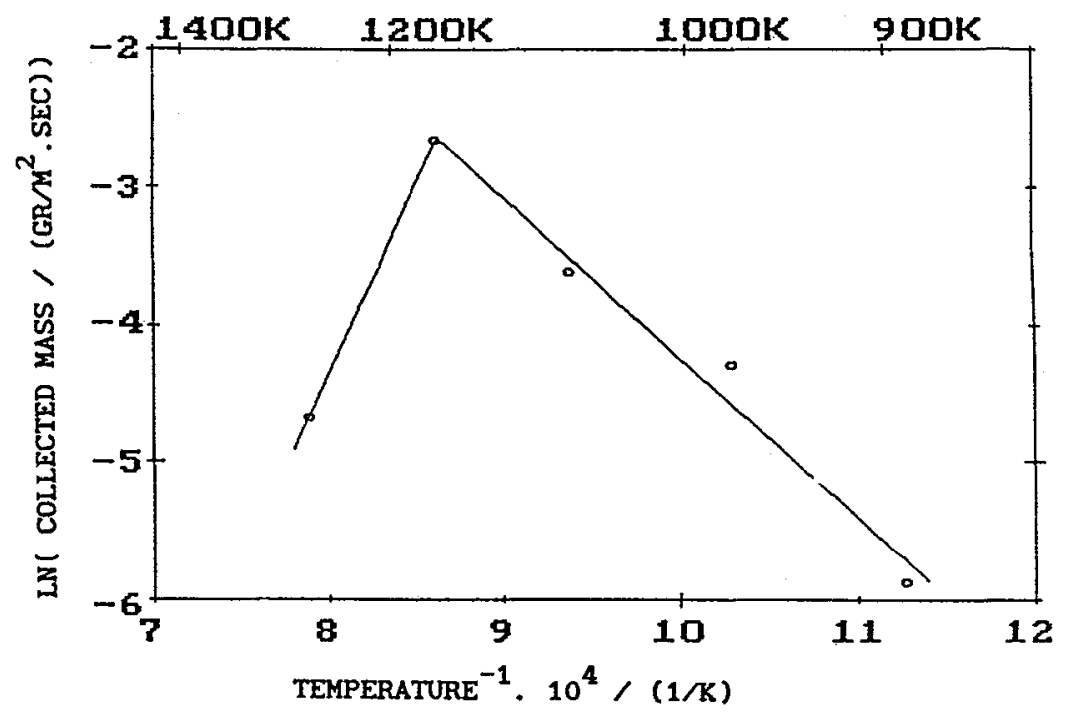

Fig. 1. Arrhenius plot of the collected TiN powder on the cooled sheet.

The primary particle diameter as determined from TEM micrographs shows a pronounced difference at high temperature in comparison with the particle diameter as determined from SF3 and QELS measurements as can be seen in Figure 2. The differences in size calculated from weight (SF3) and hydrodynamic diameter (QELS) fall well within the error of these two measurement techniques [5]. Figure 3 shows TEM micrographs of clusters formed at different temperatures.

The mass distribution as calculated from SF3 measurements shows a change from a monodisperse powder at low temperatures to a bimodal distribution at higher temperatures as can be seen in Figure 4 .

Hardly any correlation exists between reactant concentration or reactant ratio and particle size. Only at low titanium tetrachloride concentration $(0.5 \%)$ and low ammonia over titanium tetrachloride ratio $(<2)$ are the particles significantiy smaller. At low gas flow rates $(0.5 \mathrm{~cm} / \mathrm{sec})$ the powder formed consists primarily of single crystals whereas at higher gas flow rates the particles became larger and were polycrystalline. 


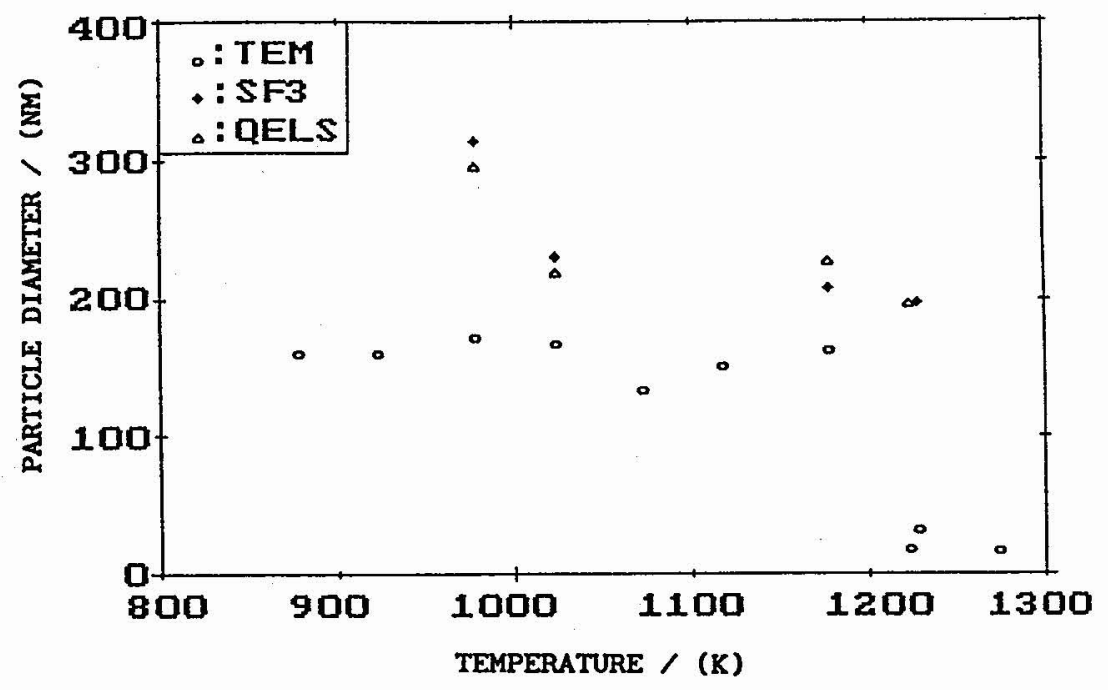

Fig. 2 Particle diameter as determined from SF3, QELS and TEM analysis.

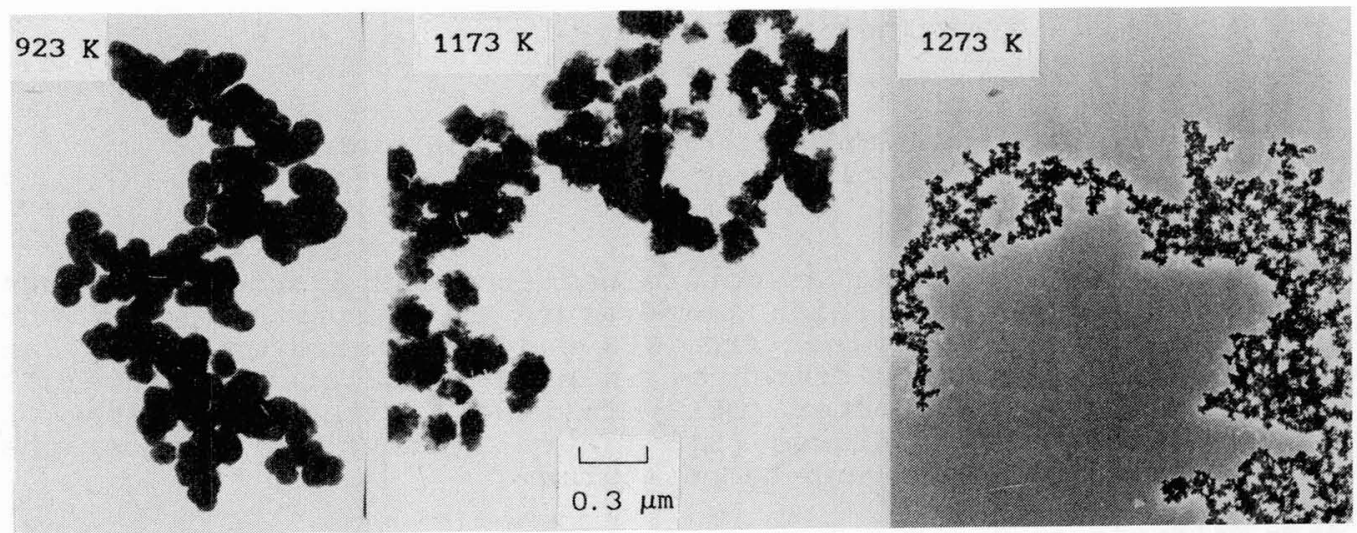

Fig. 3 TEM micrographs of TiN powder formed at different temperatures. 


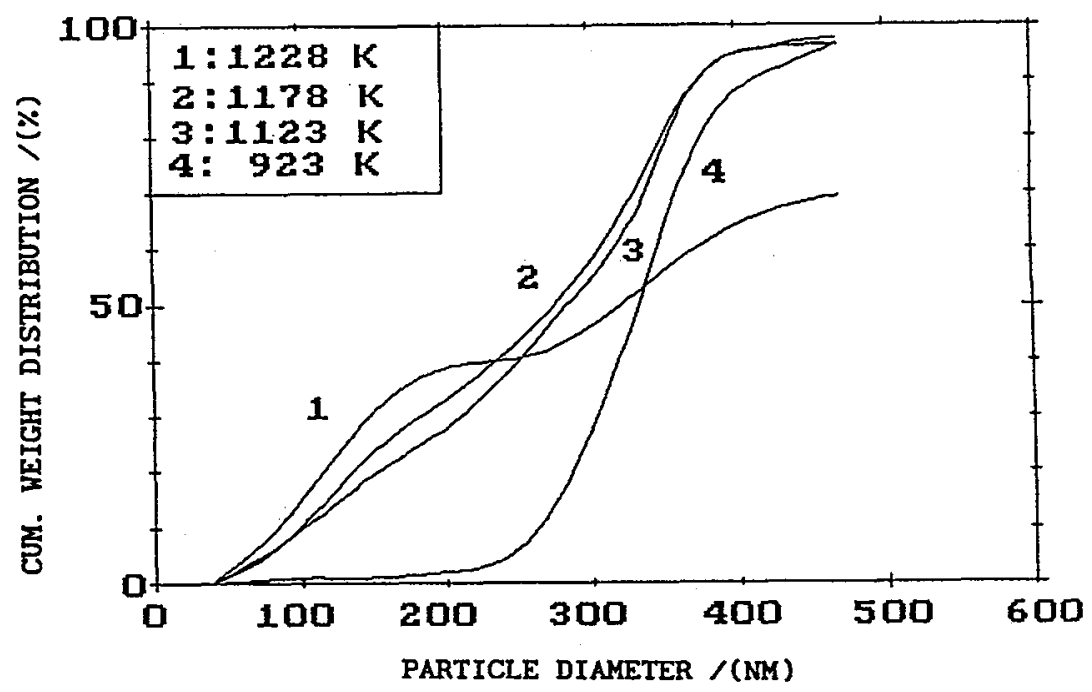

Fig. 4 Mass distribution of the TiN powder as determined from SF3.

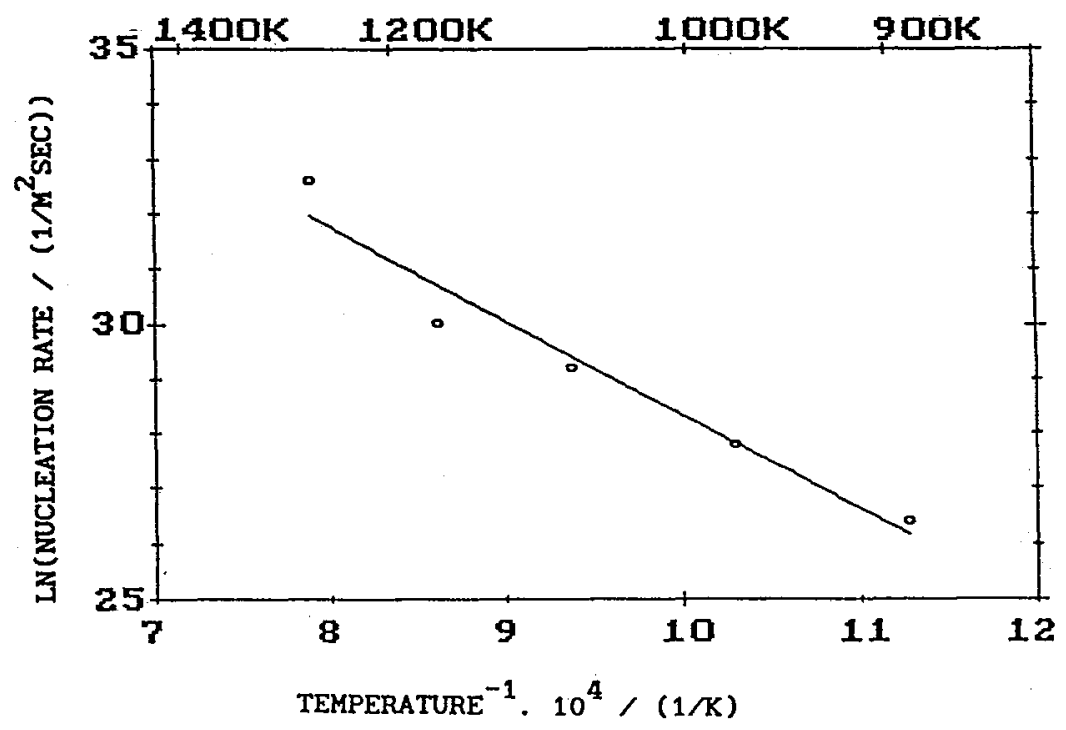

Fig. 5. Arrhenius plot of the nucleation rate of TiN powder. 
The nucleation rate is proportional to the number of the primary particles collected on the cooled molybdenum substrate. The ratio of primary particle volume as determined from transmission micrograph analysis and collected mass can be plotted in an Arrhenius plot which. yields a nucleation activation energy of $142 \pm 17 \mathrm{~kJ} / \mathrm{mole}$ as can be seen in Figure 5 .

The conversion degree is determined by the nucleation rate and growth rate of the particles. At low temperature the process is determined by the growth rate resulting in a monodisperse powder. At high temperatures the growth rate decreases and particles become agglomerated with increasing temperature resulting in a bimodal distribution, the conversion drops due to the low growth rate whereas the nucleation rate is high yielding small particles.

The sudden decrease of particle growth rate at temperatures above 1200 $K$ suggests a change of growth mechanism. At this temperature the calculated equilibrium gas phase concentration of titanium tetrachloride drops below that of titanium trichloride. The homogeneous thermodynamic equilibrium as a function of temperature is calculated with a modified version of the SoLGAS-MIX software package [7]. All the titanium, chloride, nitrogen and hydrogen containing species present in the JANAF tables [8] were taken into account. The other equilibrium pressures of metal containing compounds were negligible. This change in concentrations could conceivably be a thermodynamic reason for a change in reaction mechanism with a concomitant drop in growth rate at elevated temperatures. The kinetics of the reaction however are insufficiently known at present to exclude an acceptable mechanism. Another explanation which cannot be excluded at this stage could be the increased rate at high temperatures of a side reaction which decomposes one of the reactants necessary for growth. Particles formed at higher temperatures become less faceted and have an increasing surface area. These nuclei having a high surface roughness could be efficient catalysts for decomposition of ammonia to nitrogen and hydrogen which, apart from removing vital reactant, screens the nuclei from the gaseous titanium species, thus inhibiting growth. This mechanism does not preclude efficient nucleation and is therefore also consistent with our results. Further research to elucidate the mechanism of this decrease is in progress.

\section{CONCLUSION.}

The reaction temperature has a strong influence on the powder properties. At temperatures between $900 \mathrm{~K}$ and $1200 \mathrm{~K}$ the powder is monodisperse and is not agglomerated. The PP-CVD temperature must be chosen within this temperature regime depending on the heterogeneous growth rate necessary to fill the pores between the precipitated particles. 
REFERENCES.

[1] A. Fuchs, "The Mechanics of Aerosols", rev. ed. Pergamon, Elmsford, New York (1964).

[2] J.B. Macchesney, P.B. O'Connor, F.V.Dimarcello, J.R. Simpson and P.D. Lazay in Proceedings of 10th International Congress on Glass, 6 ceramics Society, Japan (1974) 40 .

[3] H. Komijama, T. Osawa, Y Shimogaki, N. Wakita, M. Minamiyama and T. Ueoka in Proceedings of loth International Conference on CVD, The Electrochemical Society (1987) 1119 .

[4] A. Kato, J. Hojo and Y. Okabe, Memoirs of the Faculty of Engineering, Kyushi University, 41/4 (1981) 319.

[5] B. Scarlett, H.G. Merkus, Y. Mori and J. Schoonman in Particle Size Analysis, P.J. Lloyd ed., John Wiley \& Sons Ltd., New York (1988) 107 .

[6] B.E. Dahneke ed., Measurement of Suspended Particles by Quasi-Elastic Light Scattering, John Wiley \& Sons Ltd., New York (1983).

[7] G.Erikson, Acta Chem. Scand. $27 / 7$ (1971) 2657.

[8] M.W. Chase, Jr., C.A. Davis, J.R. Downey, Jr., D.R. Frurip, R.A. McDonald and A.N. Syverud, J. Phys. Ref. Data, 14 (1985), part $1 / 2$. 